\title{
Performance Comparison of LS, LMMSE and Adaptive Averaging Channel Estimation (AACE) for DVB-T2
}

\author{
Spiridon Zettas, Pavlos. I. Lazaridis, Zaharias D. Zaharis, Stylianos Kasampalis, Neeli \\ Prasad, Ian A. Glover, and, John. P. Cosmas
}

\begin{abstract}
In this paper the performance of the Adaptive Averaging Channel Estimator (AACE-LS) which is a modified Least Square (LS) estimator and the AACE-LMMSE which is a modified Linear Minimum Mean Error (LMMSE) estimator, are compared with respect to the conventional LS and the LMMSE estimators. The AACE is an estimator which is based on the averaging of the last $N$ Scattered Pilots (SP) from the DVB-T2 model carried in the received OFDM symbols. The proposed method could in general be applied to any pilot based estimator. The noise introduced by the channel is considered as Additive White Gaussian Noise (AWGN) with zero mean and thus an averaging process is used to eliminate it. The estimator adaptively follows the fluctuations of the amplitude envelope in the time domain and adapts the size of the buffer $N$, with respect to the coherence time (Tc). Finally, based on the averaged estimated channel, the LS or the LMMSE equalizer is applied to the received signal in the frequency domain. Simulations clearly show that the performance of the AACE-LS is superior to the conventional LS estimator and is near to the performance of the LMMSE with no need of a prior knowledge of the statistics and the noise of the channel and thus if the channel is unknown to the receiver, the AACE is a good choice.
\end{abstract}

Index Terms-AWGN, channel estimation, Doppler shift, DVB-T2, LMMSE, LS, MSE, Rayleigh, OFDM, scattered pilots.

\section{INTRODUCTION}

$\mathrm{T}$ HE INCREASING demands for high data rates in modern radio communication lead both researchers and industries to adopt cutting edge technologies and techniques in order to satisfy these needs. The use of Orthogonal Frequency Division Multiplexing (OFDM) [1-2], helps to increase the period of each transmitted symbol while the overall data rate is a multiple of the number of subcarriers in each OFDM symbol. Digital Video Broadcasting Second Generation Terrestrial (DVB-T2) [3] which was standardized in 2009 and supports high data rates [4], adopted this modulation. The use of advanced channel estimators helps to compensate for the distortions that the channel is introducing.

Spiridon Zettas, Stylianos Kasampalis, and John Cosmas are with the School of Engineering and Design, Brunel University, London UB8 3PH, UK.

Pavlos I. Lazaridis is with the Department of Computing and Engineering, University of Huddersfield, HD1 3DH, UK, and ATEI of Thessaloniki.

Ian A. Glover is with the Department of Computing and Engineering, University of Huddersfield, HD1 3DH, UK.

Zaharias D. Zaharis is with the Telecommunications Centre, Aristotle University of Thessaloniki, GR-54124 Thessaloniki, Greece.

Neeli Prasad is with Aalborg University, CTiF, Aalborg, Denmark.
The channel varies in the time-domain because of the multipath fading and the relative movement of the transmitter and the receiver or because of reflectors in the signal's path. The time interval, where the channel can be assumed constant, is the coherence time $T_{c}$ and it is related to the Doppler spread. The most common problems in channel estimation are the estimation of the Doppler shift (DS), the choice of the pilot arrangement and the choice of an estimator with low complexity and high performance. The DS can be estimated with several methods. The Phase Difference of the received pilots in Rayleigh fading channels was studied in [5]. Another method was proposed in [6-7], where the variations in time of the logarithmically compressed amplitude of the received signal are measured. The autocorrelation function $R_{n}$ [8-9] of the received signal is the basis for DS estimation in several other methods. The Zero Level Crossing (ZLC) of the autocorrelation function of the received signal as a method for DS estimation is proposed in [10-11]. A comparative performance analysis of DS estimators can be found in [12].

There are two main methods for channel estimation. In the first method pilots are used, which are tones within the OFDM symbols that are known to the receiver. The second method, named blind estimation, manipulates the statistical or structural properties of the signal, and thus no pilots are needed, thus increasing the system's throughput. In [13], the performance of blind estimation is studied and an optimized algorithm is suggested. In Pilot Symbol Assisted Modulation (PSAM) [14-18] there are different pilot arrangements, the block type and the comb-type. In the block type the pilots are inserted in the same subcarrier for all OFDM symbols (time domain) or all subcarriers are pilots in every few OFDM symbols (frequency domain). This is useful for slowly timevarying channels. In fast time-varying channels the comb-type is preferred [19-21]. In the case of DVB-T2, there are both types of pilot arrangements. Block type pilots, such as Edge Pilots, Continual and Frame Closing Pilots and comb-type pilots named Scattered Pilots (SP) in 8 patterns, [3]. The choice of SP pattern is based on the channel's conditions and makes DVB-T2 robust against fading degradation.

These pilot types can be based on Least Squares (LS), Modified LS, Minimum Mean Squared Error (MMSE), and Modified MMSE, and a frequency interpolation has to be performed, [22]. The LS estimators are of low complexity and computational load but provide poor Bit Error Rate (BER) and Mean Square Error (MSE) performance compared to MMSE. MMSE offers better BER and MSE in exchange for high 
complexity, computational load and the requirement to know in advance the channel's second order statistics. In [23] a comparison of Maximum Likelihood Estimator (MLE) and the Bayesian Minimum Mean Square Error Estimator (MMSEE) is given. In [24] a modified MMSE estimator, where only the taps with significant energy are considered is presented, and in [25] another modified MMSE estimator is considered based on Singular Value Decomposition (SVD). In this paper the Adaptive Averaging Channel Estimator AACE-LS [26-28], which is a modified LS estimator, is compared with the conventional LS and the LMMSE estimator. The AACEMMSE which is an AACE in conjunction with LMMSE is also tested. First, the coherence time $T_{c}$ is derived based on Doppler Shift estimation. Then, the pilots SP of the OFDM symbols that were received within the coherence time are interpolated and averaged. Assuming that the noise introduced by the channel is Additive White Gaussian Noise (AWGN) with zero mean and variance $\sigma^{2}, N \sim\left(0, \sigma^{2}\right)$, the averaging process eliminates the noise and makes the estimation easier and more accurate.

The rest of this paper is organized as follows: Section II describes the system model, is Section III the Doppler Shift estimation is analyzed, in Section IV the AACE is explained, in Section $\mathrm{V}$ the simulations and the results in BER vs SNR and MSE vs SNR curves are presented, finally in Section VI conclusions and future work are provided.

\section{System Model}

The OFDM mathematical expression is given in (1):

$$
\begin{gathered}
\mathrm{x}(t)=\sum_{n=-\infty}^{\infty} \sum_{k=0}^{N-1} c_{n, k} \cdot g_{k}\left(t-n T_{s}\right) \\
\text { with } g_{k}(t)= \begin{cases}e^{i 2 \pi f_{k} t}, & \mathrm{t} \in\left[0, \mathrm{~T}_{s}\right] \\
0, & \text { elsewhere }\end{cases} \\
\text { and } f_{k}=f_{0}+\frac{k}{T_{s}}, \quad k=0,1, \ldots, N-1
\end{gathered}
$$

where, the symbol of the $k$-th subcarrier of the $n$-th OFDM symbol is denoted as $c_{n, k}, f_{0}$ is the starting (lowest) frequency and $f_{k}$ is the frequency of the $k$-th subcarrier.

In order to eliminate the Inter Carrier Interference (ICI) and the Inter Symbol Interference (ISI) a cyclic prefix, as Guard Interval (GI) is used. The available values of the GI in DVBT2 are $1 / 4,19 / 128,1 / 8,19 / 256,1 / 16,1 / 32$, and $1 / 128$.

The channel is assumed as flat Rayleigh fading channel, which implies that the subcarriers of the OFDM symbol suffer of the same magnitude of fading. The channel's envelope follows the Rayleigh distribution with a power density function (pdf) equal to (2):

$$
\mathrm{f}(\mathrm{x}, \sigma)=\frac{x}{\sigma^{2}} \mathrm{e}^{-x^{2} /\left(2 \sigma^{2}\right)}, x \geq 0
$$

where $\sigma$ is the standard deviation.
The Doppler spectrum $\mathrm{S}(\mathrm{v})$ as a function of the Doppler frequency shift $v[29]$ is given in (3):

$$
S(v)=\frac{1}{\pi f_{D} \sqrt{1-\left(\frac{v-f_{c}}{f_{D}}\right)^{2}}}
$$

where $f_{c}$ is the carrier frequency and $f_{D}$ is the Doppler Spread (DS).

\section{DOPPLER SHIFT ESTIMATION}

The Doppler Spread (DS) introduced by the channel is a factor indicating the typical fading rate of the channel in the time domain. In the rest of the paper the channel will be assumed as Rayleigh. In [6-7] the Logarithmic Envelope (LE) autocorrelation of the received signal is considered and a simple expression of the estimated Doppler frequency $\hat{f}_{D}$ is derived. The squared deviation $V$ of the logarithmically compressed envelope $y_{i}=20 \log _{10}\left(x_{i}(t)\right)$, of the received signal $x_{i}(t)$, sampled with period $\tau$, is computed for the last $N$ considered samples:

$$
V=\frac{1}{N} \sum_{t=0}^{N-1}\left(y_{i+1}-y\right)^{2}
$$

Then, the estimation of Doppler frequency $\hat{f}_{D}$ can be obtained as (5) according to [6], or as (6) according to [7]:

$$
\begin{gathered}
\hat{f}_{D}=\left\{\begin{array}{lr}
15.25 \sqrt{V}, & V<3.8 \\
4.033 V+19.60, & V \geq 3.8
\end{array}\right. \\
\hat{f}_{D}=\left\{\begin{array}{lr}
\frac{(V+0.796)}{41.664 \pi}, & V \leq 1.087 \\
\frac{(V+2.120)}{58.302 \pi}, & 1.087<V \leq 4.02 \\
\frac{(V+4.789)}{77.3 \pi}, & V>4.002
\end{array}\right.
\end{gathered}
$$

Based on Clarke's model [8-9] the autocorrelation function $R(z)$ can expressed as in (7):

$$
R(\mathrm{z})=J_{0}\left(2 \pi f_{D} z T_{s}\right)
$$

Where, $z=n \cdot T s, n$ is the index of the $n^{\text {th }}$ received OFDM symbol and $T_{s}$ is the OFDM symbol duration and $J_{0}(\cdot)$ is the Bessel function of zero order and of the first kind. Interpolating in (8) the first index $z$ where is $R(z)<0$ and $z-1$ where $R(z-1)>0$ we get $\hat{z}_{0}$ which is where $R\left(\hat{z}_{0}\right) \cong 0$ :

$$
\hat{z}_{0}=\frac{\mathrm{R}(z)}{\mathrm{R}(z-1)-R(z)}+z
$$

But, we have:

$$
J_{0}(2.4048255577)=0
$$

Combining (7), (9) we get the formula for $\hat{f}_{D}$ as: 


$$
\hat{f}_{D}=\frac{2.4048255577}{2 \pi \cdot \hat{z}_{0} \cdot T_{s}}
$$

\section{Adaptive AVERAging Channel Estimator}

The problem of channel estimation is to estimate the channel's frequency response $\widehat{H}_{l, k}$ of the $l^{\text {th }}$ subcarrier in the $k_{t h}$ OFDM symbol and then to multiply the inverse $\widehat{H}_{l, k}^{-1}$ and the received signal $Y_{l, k}$ in order to estimate the transmitted signal $\hat{X}_{l, k}$. In channel estimation the error $E\left\{H_{l, k}-\widehat{H}_{l, k}^{-1}\right\}$ should be equal to zero. The problem is that the channel's frequency response $H_{l, k}$ cannot be perfectly estimated.

\section{1) LS estimator}

The LS estimator minimizes the cost function $E(11)$ :

$$
E=\min \left\{(Y-\hat{H} X)^{H} \cdot(Y-\hat{H} X)\right\}
$$

where, $\quad Y=\left[Y_{0}, Y_{1}, \ldots, Y_{K-1}\right]^{T}, H=\left[H_{0}, H_{1}, \ldots, H_{K-1}\right]$, $X=\left[X_{0}, X_{1}, \ldots, X_{K-1}\right]^{T},(\cdot)^{T}$ denotes the matrix transpose and the (. $)^{H}$ denotes the Hermitian transpose. The relation between the received signal $Y$, the transmitted signal $X$, the channel frequency response $H$ and the Additive white Gaussian Noise (AWGN) $N$ is given in matrix notation in (12):

$$
Y=H \cdot X+N
$$

The estimated channel's frequency response on the scattered pilots of the DVB-T2 system $\hat{H}_{L S}{ }^{P}$ after the noise elimination using the proposed averaging process, is given in (13):

$$
\hat{H}_{L S}^{P}=Y \cdot\left(X^{P}\right)^{-1}
$$

After interpolation of the $\hat{H}_{L S}{ }^{P}$, the estimation of $\hat{H}_{L S}$ is derived.In case of LS the Mean Squared Error (MSE) of the channel estimation is given in (14):

$$
M S E_{L S}=\left(H-\hat{H}_{L S}\right)^{H} \cdot\left(H-\hat{H}_{L S}\right)
$$

\section{2) LMMSE estimator}

The implementation of an estimator that satisfies the MMSE criterion which eliminates the noise interference, is unfeasible to be implemented in practice. A simplified Linear Minimum Mean Squared Error is proposed in [25] and formulated as in (13):

$$
H_{L M M S E}=R_{H H}\left(R_{H H}+\frac{\beta}{S N R} I\right)^{-1} \cdot \hat{H}_{L S}
$$

where: $R_{H H}=E\left\{H H^{T}\right\}$ is the autocorrelation matrix of the channel, $\beta=E\left\{\left|X_{k}\right|^{2}\right\} E\left\{\left|1 / X_{k}\right|\right\}^{2}$ is a constant related to the chosen QAM constellation diagram (for 16-QAM, $\beta=17 / 9$ ), the Signal to Noise Ratio (SNR) is expressed as $S N R$ $=E\left\{\left|X_{k}\right| 2\right\} / \delta_{n}{ }^{2}$ and $\delta_{\mathrm{n}}{ }^{2}$ denotes the variance of noise $E\left\{\left|n_{k}\right|^{2}\right\}$.

The Mean Squared Error (MSE) in case of LMMSE is given in (16):

$$
M S E_{\text {LMMSE }}=\left(H-\hat{H}_{L M M S E}\right)^{H} \cdot\left(H-\hat{H}_{\text {LMMSE }}\right)
$$

A detailed description of the Adaptive Averaging Channel Estimator (AACE) can be found in [26]. The coherence time $T c$, which is the time interval where the channel is assumed as flat, has to be larger than the buffering time $T_{B}=n T_{s}$, where $n$ is number of the buffered OFDM symbols and $T_{S}$ is the OFDM symbol's duration. The coherence time $T_{C}$ is related to $f_{D}$ with (17), [27]:

$$
T_{c}=\sqrt{\frac{9}{16 \pi \hat{f}_{D}}}=\frac{0.423}{\hat{f}_{D}}
$$

where, $\hat{f}_{D}$ is the Doppler Spread derived from (10). Finally, the averaging process, [24], is mathematically expressed in (18):

$$
H_{L S}^{P}(\mathrm{n})=\frac{1}{P_{n}} \sum_{i=1}^{B} \operatorname{Buffer}(\mathrm{i}, \mathrm{n})
$$

where, $P n$ is the number of pilots in a specific subcarrier for the last B buffered OFDM symbols into the Buffer $(B x N)$.

\section{Simulations And Results}

This section demonstrates the performance analysis of the conventional LS, the proposed AACE-LS, the LMMSE, and the AACE-MMSE. The comparison is based on BER vs SNR curves, and MSE vs SNR curves.

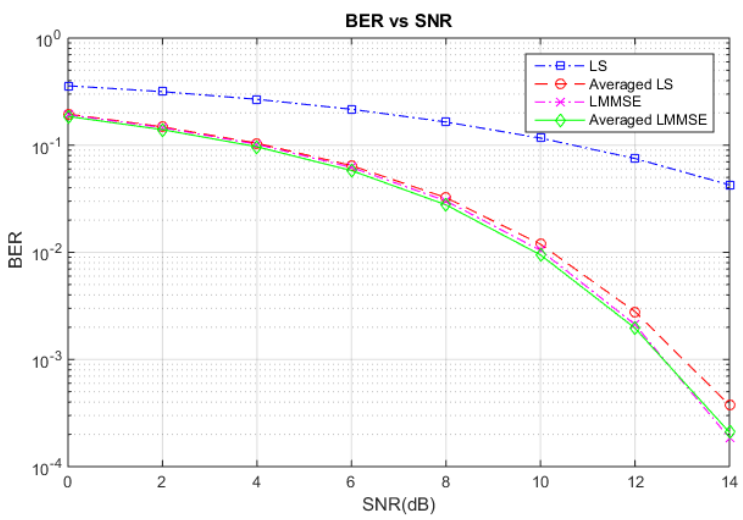

Fig. 1. BER for Doppler frequency $f_{d}=2 \mathrm{~Hz}$

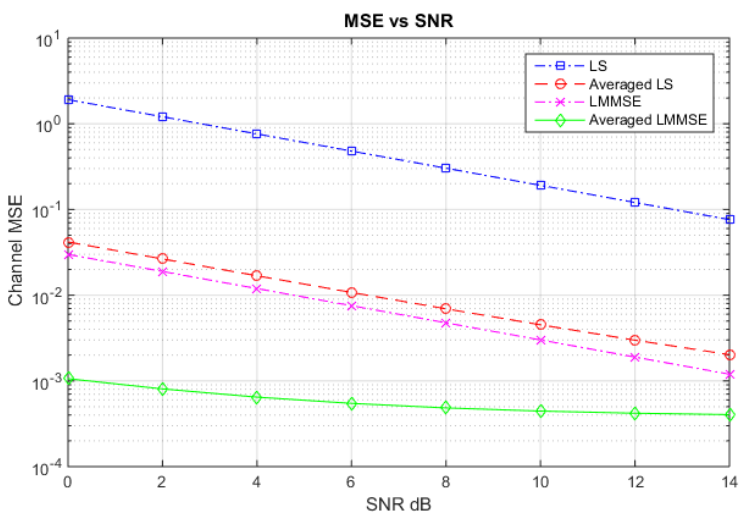

Fig. 2. MSE for Doppler frequency shift $f_{d}=2 \mathrm{~Hz}$.

For small Doppler frequency shifts, $f_{D}=2 \mathrm{~Hz}$, the superiority of AACE-LS against conventional LS is depicted in both BER and MSE curves. In Fig. 1 the performance of AACE-LS is 
very close to the performance of LMMSE and in Fig. 2 the MSE for the AACE-LS is very close to the LMMSE.

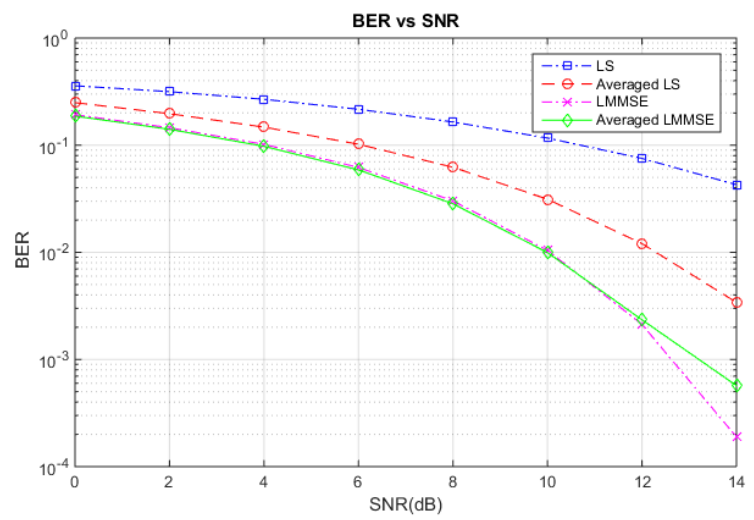

Fig. 3. BER for Doppler frequency shift $f_{d}=15 \mathrm{~Hz}$.

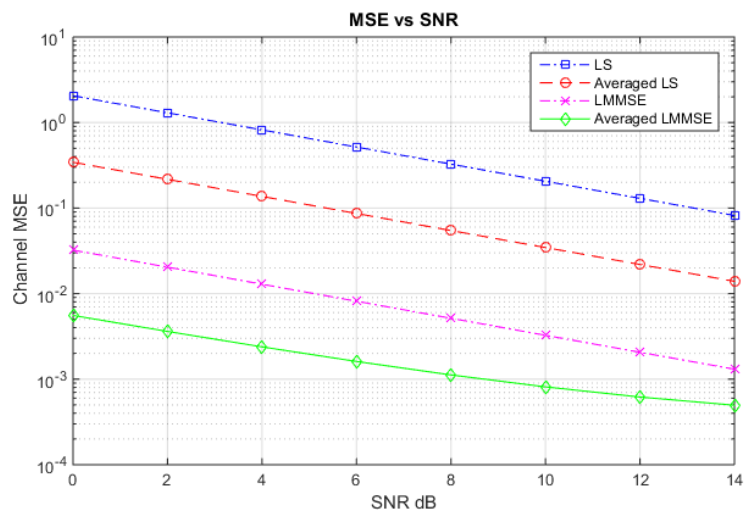

Fig. 4. MSE for Doppler frequency shift $f_{d}=15 \mathrm{~Hz}$.

In case of a higher Doppler frequency shift, $f_{D}=15 \mathrm{~Hz}$, the performance of AACE-LS is slightly degraded as Fig. 3 depicts and is in the middle of the conventional LS and the LMMSE. The MSE of AACE-LS is also degraded and it is in the middle of the LS and LMMSE curves as Fig.4 illustrates.

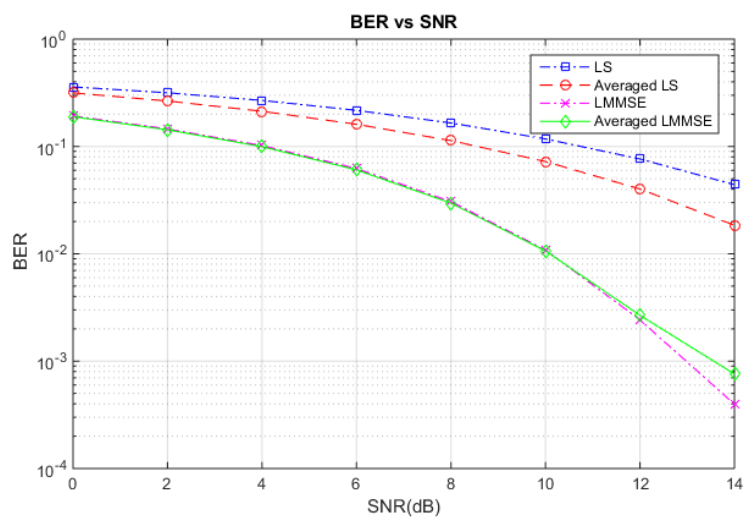

Fig. 5. BER for Doppler frequency shift $f_{d}=50 \mathrm{~Hz}$.

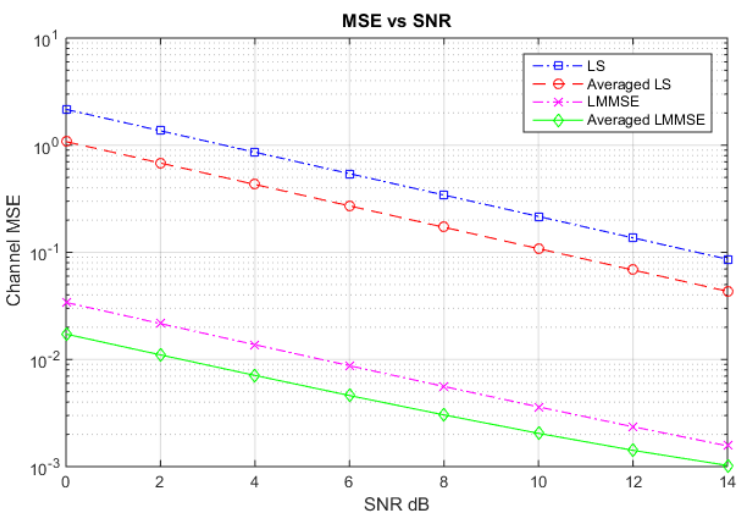

Fig. 6. MSE for Doppler frequency shift $f_{d}=50 \mathrm{~Hz}$

As the Doppler Shifts inceases the performance of the AACE-LS degrades further and converges to the performance of the LS. For Doppler Shifts of $f_{D}=50 \mathrm{~Hz}$ the BER performance of the AACE-LS is depicted in Fig. 5 and the MSE in Fig. 6.

From the BER vs SNR point of view, in all scenarios provided, the performance of the AACE-LMMSE is similar to LMMSE and thus the implementaion of AACE with LMMSE is meaningless.

\section{CONClusions AND Future Work}

The performance of AACE-LS and AACE-LMMSE estimators were studied in this paper. The simulations in MATLAB clearly show that the AACE-LS estimator has better performance compared with the conventional LS estimator especially for low Doppler shifts. The implementation of AACE with LMMSE leads to negligible improvement compared with the conventionasl LMMSE and thus it provides no extra benefits. Consequently, if the receiver has low computational resources and/or the channel's statistics are unknown, then the AACE-LS estimator is a possible choice. Future works include performing simulations for Multiple Input Multiple Output (MIMO) systems.

\section{ACKNOWLEDGMENT}

Parts of this work were performed within the NATO SfP984409 project "Optimization and Rational Use of Wireless Communications Bands" (ORCA). The authors would like to thank everyone involved.

\section{REFERENCES}

[1] R. Prasad, "OFDM for Wireless Communication Systems". Artech House, 2004.

[2] Zou, W.Y.; Yiyan Wu, "COFDM: an overview," Broadcasting, IEEE Transactions on, vol.41, no.1, pp.1-8, Mar 1995.

[3] Digital Video Broadcasting (DVB). Frame structure channel coding and modulation for a second generation digital terrestrial television broadcasting system (DVB-T2), ETSI EN 302755 V1.3.1, Apr. 2012.

[4] Digital Video Broadcasting, Fact Sheet, 2nd Generation Terrestrial, DVB-T2, Aug. 2014. Available: https://www.dvb.org/resources/public/factsheets/dvb-t2 factsheet.pdf

[5] Hadiansyah, W.M., Suryani, T., Hendrantoro, G., "Doppler spread estimation for OFDM systems using Phase Difference method in 
Rayleigh fading channels" Telecommunication Systems, Services, and Applications (TSSA), 2012 7th International Conference on, pp.147-152, 30-31 Oct. 2012.

[6] Holtzman J.M., Sampath A., "Adaptive averaging methodology for handoffs in cellular systems," Vehicular Technology, IEEE Transactions on , vol.44, no.1, pp.59-66, Feb. 1995.

[7] Sui Dan, Hu Hanying, "A novel Doppler shift estimator based on LE algorithm in mobile communication systems," ITS Telecommunications Proceedings, 2006 6th International Conference on, pp. 553-556, 21-23 June 2006.

[8] W. C. Jakes, Ed., Microwave Mobile communications, New Jersey: IEEE Press, 1993.

[9] Clarke, R.H., "A statistical theory of mobile-radio reception," Bell System Technical Journal, The, vol.47, no.6, pp.957-1000, July-Aug. 1968.

[10] Won-Gyu Song, Jong-Tae Lim, "Pilot-symbol aided channel estimation for OFDM with fast fading channels," Broadcasting, IEEE Transactionson, vol.49, no.4, pp.398-402, Dec. 2003.

[11] Singhapan A., Naito K., Mori K., Boonsrimuang P., Kobayashi H., "Doppler frequency spread estimation for OFDM systems in time-varying fading channel," Electrical Engineering/Electronics, Computer, Telecommunications and Information Technology (ECTI-CON), 2012 9th International Conference on, vol., pp.1-4, 16-18 May 2012.

[12] Tepedelenlioglu, C., "Performance analysis of velocity (Doppler) estimators in mobile communications," Acoustics, Speech, and Signal Processing (ICASSP), 2002 IEEE International Conference on , vol.3, no., pp.III-2201,III-2204, 13-17 May 2002

[13] Martínez, L.; Robert, J.; Meuel, H.; Sobrón, I.; Mendicute, M.; , "Improved robustness for channel estimation without pilots for DVBT2," Broadband Multimedia Systems and Broadcasting (BMSB), 2010 IEEE International Symposium on, vol., no., pp.1-5, 24-26 Mar. 2010.

[14] Cavers, J.K., "An analysis of pilot symbol assisted modulation for Rayleigh fading channels [mobile radio]," Vehicular Technology, IEEE Transactions on, vol.40, no.4, pp.686-693, Nov. 1991.

[15] Ye Li, "Pilot-symbol-aided channel estimation for OFDM in wireless systems," Vehicular Technology Conference, 1999 IEEE 49th, vol.2, no., pp.1131-1135 vol.2, Jul. 1999

[16] Tsatsanis, M.K.; Zhengyuan Xu, "Pilot symbol assisted modulation in frequency selective fading wireless channels," Signal Processing, IEEE Transactions on, vol.48, no.8, pp.2353-2365, Aug. 2000.

[17] Xiaodong Cai; Giannakis, G.B., "Adaptive PSAM accounting for channel estimation and prediction errors," Wireless Communications, IEEE Transactions on , vol.4, no.1, pp.246,- 56, Jan. 2005.

[18] Zijian Tang; Cannizzaro, R.C.; Leus, G.; Banelli, P., "Pilot-Assisted Time-Varying Channel Estimation for OFDM Systems," Signal Processing, IEEE Transactions on , vol.55, no.5, pp.2226-2238, May 2007.

[19] Meng-Han Hsieh; Che-Ho Wei,, "Channel estimation for OFDM systems based on comb-type pilot arrangement in frequency selective fading channels," Consumer Electronics, IEEE Transactions on, vol.44, no.1, pp.217-225, Feb 1998 .

[20] Yushi Shen and Ed Martinez, "Channel Estimation in OFDM Systems", Free scale Semiconductor, AN3059.

[21] Coleri, S.; Ergen, M.; Puri, A.; Bahai, A., "Channel estimation techniques based on pilot arrangement in OFDM systems,"Broadcasting, IEEE Transactions on, vol.48, no.3, pp.223-229, Sep. 2002

[22] Tomasin, S.; Butussi, M., "Analysis of interpolated channel estimation for mobile OFDM systems," Communications, IEEE Transactions on , vol.58, no.5, pp.1578-1588, May 2010

[23] Morelli, M.; Mengali, U., "A comparison of pilot-aided channel estimation methods for OFDM systems," Signal Processing, IEEE Transactions on, vol.49, no.12, pp.3065-3073, Dec 2001

[24] Van de Beek, J.-J.; Edfors, O.; Sandell, M.; Wilson, S.K.; Ola Borjesson, P., "On channel estimation in OFDM systems," Vehicular Technology Conference, IEEE 45th, vol.2, no., pp.815-819, vol.2, 25-28 Jul. 1995.

[25] Edfors, O.; Sandell, M.; Van de Beek, J.-J.; Wilson, S.K.; Borjesson, P.O., "OFDM channel estimation by singular value decomposition," Communications, IEEE Transactions on, vol.46, no.7, pp.931-939, Jul. 1998.

[26] Zettas, S.; Lazaridis, P.I.; Zaharis, Z.D.; Kasampalis, S.; Cosmas, J., "A pilot aided averaging channel estimator for DVB-T2," Broadband Multimedia Systems and Broadcasting (BMSB), 2013 IEEE International Symposium on, vol., no., pp.1-8, 5-7 June 2013.
[27] Zettas, S.; Kasampalis, S.; Lazaridis, P.; Zaharis, Z.D.; Cosmas, J., "Channel estimation for OFDM systems based on a time domain pilot averaging scheme," Wireless Personal Multimedia Communications (WPMC), 2013 16th International Symposium on , vol., no., pp.1,6, 2427 June 2013.

[28] Zettas, S.; Lazaridis, P.I.; Zaharis, Z.D; Kasampalis, S.; Cosmas, J., "Adaptive averaging channel estimation for DVB-T2 using Doppler Shift information," Broadband Multimedia Systems and Broadcasting (BMSB), 2014 IEEE International Symposium on, vol., no., pp.1,6, 2527 June 2014.

[29] B. Sklar, Digital Communications, Fundamentals and Applications, $2^{\text {nd }}$ Ed. New Jersey, USA: Prentice Hall, 2006, pp. 969. 